
\title{
R Reserach S Suare \\ The biceps challenge - an experimental approach to the anterior shoulder pain, indicating a new treatment logarithm
}

\author{
Kristian Sørland Humborstad \\ Helse Forde HF \\ Jonn Terje Geitung ( $\boldsymbol{\nabla}$ j.t.geitung@medisin.uio.no ) \\ https://orcid.org/0000-0001-9259-1060
}

\section{Research article}

Keywords: Biceps tendon; MRI; tenodesis, shoulder pain

Posted Date: October 16th, 2019

DOI: https://doi.org/10.21203/rs.2.16115/v1

License: (c) (1) This work is licensed under a Creative Commons Attribution 4.0 International License.

Read Full License 


\section{Abstract}

Abstract Background The biceps muscle's long head (LHB) with its tendon and origin represents the most important cause of anterior shoulder pain. Conservative treatments (non-steroid antiinflammatory drugs, injections with steroids and physiotherapy) will normally be the first line of treatment. Long-standing pain will usually lead to surgical treatment with either tenodesis or tenotomy as alternative procedures. The main aim of our investigation was to find structural changes that may change the surgical treatment logarithm in these patients. Secondary aims were 1) to see if experimental settings would better visualize such changes on MRI, and correlate this to histological examinations. 2) to see if visualized changes to histology and MRI would have significant correlations to clinical findings and surgical practice. Methods The patients were evaluated at 1) the first outpatient contacts with the orthopedic surgeon, 2) preoperative 1.5-Tesla MRI, 3) per-op findings with arthroscopy, 4) experimental 7-Tesla MRI, and 5) histology with standard $\mathrm{H}+\mathrm{E}-$ staining. Findings Our experimental study showed that all the degenerative changes in the LHB-tendon were in the intraarticular part of the tendon, not in the intertubercular sulcus part (shown clearly at the experimental work). None of the preoperative 1.5 Tesla MRI helped us in the clinical decision-making. We did also construct a grading system of peroperative evaluation of the LHBtendon degeneration that will be useful in clinical decision-making. Interpretation Our experimental study showed a pathological-anatomical correlate to the anterior shoulder pain. In our opinion, it is important to remove the degenerated intraarticular part of the tendon, this in order to avoid postoperative inflammation, pain and prolonged sick leave. A subpectoral biceps tenodesis with removal of the proximal part of the tendon is accordingly the treatment of choice in these patients.

\section{Background}

The biceps muscle's long head (LHB) with its tendon and origin represents the most important cause of anterior shoulder pain. The pathology in the LHB is mostly degenerative and inflammatory. We often see thickening of the articular part of the tendon. Conservative treatments (non-steroid anti-inflammatory drugs, injections with steroids and physiotherapy) will normally be the first line of treatment. Longstanding pain will usually lead to surgical treatment with either tenodesis or tenotomy as alternative procedures. No study supports one surgical procedure as the superior treatment of choice $(1,2,10)$. The surgical procedure chosen depends on the surgeon's preferences and skills, the patients' age, gender, and functional demand.

Tenotomy is a simple cutting and releasing of the LHB shortly distal to the origin at the upper part of the glenoid labrum. The tendon then slips down the sulcus and will adhere by itself to the sulcus and transverse ligament, and will give pain-relief and good functional outcome in most of the patients $(8,9)$. Cosmetic changes, muscle cramps in the biceps muscle and pain in the sulcus-area are well known complications after LHB-tenotomies (4).

Tenodesis is a tenotomy, and a reinsertion of the tendon either in the intertubercular sulcus or in the subpectoral part of humerus by the use of interference-screws (5) and anchors (6). Because of the need of surgical dissection, the use of Homan retractors, drill-holes, interference-screws and anchors, there is a 
risk of postoperative pain, infection and nerve-lesion $(4,7)$ after this type of surgery, however most studies report a low rate of complications and good functional outcome after these procedures $(1,2,3)$. In the experimental study we have done both histological and 7-Tesla-MRI-scanning of the whole proximal removed LHB tendon rests after subpectoral tenodesis. The main aim of our investigation was to find structural changes that may change the surgical treatment logarithm in these patients. Secondary aims were 1) to see if experimental settings would better visualize such changes on MRI, and correlate this to histological examinations. 2) to see if visualized changes to histology and MRI would have significant correlations to clinical findings and surgical practice.

\section{Methods}

We included seven consecutive patients operated with subpectoral tenodesis, for a short period of time (6 weeks). The consent was signed on beforehand, and the Regional Ethical Committee approved the study. The age and sex differed (Table 2). The patients were referred for anterior shoulder pain. We did patient evaluation at 1) the first outpatient contacts with the orthopedic surgeon, 2) pre-op MRI, 3) per-op findings, 4) experimental MRI, and 5) histology. On clinical examination, all had a positive O'Brian test (18), Speeds test (17) and pain by palpation in the intertubercular sulcus. All had preoperative MRIscanning with 1.5-Tesla MRI, but the radiologist did not describe the LHB origin at the superior labrum and the tendon itself thoroughly. None of the patients had cuff rupture.

The procedure was done in beach chair position, and with the patient in total anaesthesia (TIVA). A diagnostic arthroscopy was done and other relevant intraarticular pathology surgically addressed. We put a stay suture in the biceps tendon approx. $20 \mathrm{~mm}$ from the tendinolabral junction and used an arthroscopic scissor to cut the tendon medial to the stay suture. We did not use a shaver or a RF (radiofrequency) probe to cut the tendon to avoid further damage to the tendon other than the clean cut. Then a small skin incision was made at the level of the lower part of the pectoralis major tendon, subcutaneous dissection down to this tendon, where the LHB tendon run on the medial side of the pectoralis major tendon. Then we pulled the LHB-tendon up with a heiss forceps, did a tenodesis using an all-thread anchor, and cut the proximal part of the tendon, usually $6 \mathrm{~cm}$ long.

At the proximal and distal part of the removed tendon, we made 2 slices, approx. $5 \mathrm{~mm}$ each, for transverse histologic examination. The rest of the tendon were examined longitudinally, with an intraarticular sone, a transitional sone and an intrasulcal sone. The tendons were put in a saline solution and brought to the experimental MRI and stored at $4 \mathrm{oC}$. On the day of scanning, the tendons were taken out of the saline, pat-dried to remove excess surface water, and then transferred into a $50 \mathrm{ml}$ plastic centrifuge tube. The tube was then filled with Fomblin oil (perfluoropolyether Y04 grade fluid, from FenS, Netherlands), which is invisible on proton MRI since hydrogen atoms in oil are replaced by fluorine. 
Fomblin oil reduces susceptibility artifacts that would appear if the tissue sample were surrounded by air.

Scanning was performed on a 7T horizontal-bore magnet using a 69-cm ID quadrature volume resonator (both from Bruker Corporation, Germany). Although the tissue cross-section was only around $1 \mathrm{~cm}$ in diameter and as such could be scanned with a smaller ID volume resonator, the samples were on average $5 \mathrm{~cm}$ long, so to guarantee homogeneous RF field along the entire sample, we had to use the volume resonator with the biggest ID. After a 3-plane localizer scan, we first performed a short (approximately 7 min) low-resolution scan to guarantee the quality of slice positioning. Afterwards, we performed a highresolution FLASH acquisition with the following imaging parameters: acquisition matrix 654x256, upsampled to $850 \times 256$, in-plane resolution $59 \mathrm{~mm} \times 59 \mathrm{~mm}$, slice thickness $5 \mathrm{~mm}$, TR/TE=400ms $/ 5.5 \mathrm{~ms}$, number of averages 80 . Total scan time was $2 \mathrm{~h}, 16 \mathrm{~min}, 32 \mathrm{sec}$. After the MRI, the histology was done at the same experimental department.

We constructed a grading system that may be used on both peroperative findings, histology and seven Tesla MRIscanning (Table 1), which were used throughout the study. We adapted this grading system to Fushs and Goutallier grading system for MRI and CT alterations in the supraspinatus muscle $(11,12)$.

Results: Our experimental study showed that all the degenerative changes in the LHB-tendon were in the intraarticular part of the tendon (I), not in the intertubercular sulcus (S). We also found in both the histological examination and the 7-Tesla MRI that the diameter of the tendon is greater in the intraarticular part than in the intertubercular sulcus part. We may thus state that the more degeneration, the greater the difference, and the greater the ratio diameter $\mathrm{I} /$ diameter $\mathrm{S}$ (Table 2). Because of this, the more degeneration of the tendon, the more wedge-shaped is the transitional zone between the intraarticular and sulcus part of the tendon.

Figure 1 illustrates changes on the histology and seven Tesla MRI-scanning in minimal, moderate and severe degeneration of the biceps tendon. The intratendinous structure is preserved in the intertubercular sulcus part of the tendon, but fibrosis ant fatty infiltration can also be seen in the grade 3 severe degenerated LHB-tendon in the intertubercular sulcus part of the tendon.

Figure 2 illustrates the changes in preoperative diagnostic 1.5 Tesla MRI, peroperative finding, histology and 7Tesla postoperative MRI scanning of one patient with grade 2 moderate degenerative changes in 
the LHB-tendon.

None of the peropertive 1.5 Tesla MRI-scannings gave us information about the LHB-tendon that we could use in the clinical decision-making.

Discussion: In our opinion, our results should alter the surgical practice in the surgical treatment of degenerative changes and pain in the LHB-tendon. Today we have two different procedures; biceps tenodesis and biceps tenotomy. Our results suggest that the subpectoral biceps tenodesis with removal of the intraarticular part of the tendon is the surgical treatment of choice when there is peroperative findings that suggest degeneration of the LHB-tendon.

We have found by our combination of MRI - and histological studies that close to all pathology in the LHB-tendon are located in the intraarticular part of the tendon, with degeneration, fibrosis and fatty infiltration. This implies that it is the intraarticular part of the tendon, and not the part situated in the sulcus that gives the patient the LHBtendon mediated shoulder pain. By doing a simple tenotomy the wedge, shaped LHB-tendon will stay in the intertubercular sulcus and cause inflammation and necrosis of the degenerated LHB-tendon, with further anterior pain in the shoulder after the surgery.

Further, on we have shown that MRI may demonstrate LHB-tendon pathology preoperatively better than it does today, and that none of the preoperative MRIs in our experimental study gave us important information of the tendon.

One of the weaknesses of our study is that we have a small material, but the strength is that all the tendons show that it is the intraarticular part of the tendon that is degenerated.

Gill et al reported pain relief in $96,7 \%$, return to sport in $90 \%$ and return to work in $96,7 \%$ after an isolated arthroscopic bicepstenotomy in a group of 30 patients, but also a complication rate of $13.3 \%$ (pain with activity, cosmetic deformity) in 4 of the 30 patients (5). Walch et al reported a $87 \%$ success rate after arthroscopic bicepstenotomy in patients with irreparable rotator cuff tears, emphasizing the role of the biceps as a pain generator in the shoulder and the ability to alleviate pain by its removal from the glenohumeral joint (8). 
Heckman et al (13) review in their article the treatment of LHB-tendon pathology and indications for revision of tenodesis and tenotomies. It is common with pain, muscle cramps and cosmetic problems after biceps tenotomies. Subpectoral bicepstenodesis and completely removing the tendon from the sulcus, as a method of revision of failed LHB-tenotomies, is the treatment of choice.

Pascal Boileau et al (14) described in 2004 a macroscopic hypertrophy of the LHB-tendon as a mechanical condition that entrap the tendon within the joint and locking the arm on elevation. The patients presented with anterior pain and loss of active and passive elevation averaging 10-20 degrees (14). Because of this hypertrophy, the tendon is unable to slide into the bicipital groove during elevation. The complete and symmetrical elevation was restored after resection of the intraarticular portion of the LHB-tendon.

Michael Joseph et al (15) did a histological and molecular characterization of the intraarticular and extraarticular portion of the post tenotomi LHB-tendon in patients with LHB-tendinopathy. The intraarticular tendon exhibited histological characteristics of tendinopathy, compared with the extra-articular portion, had structural disorganization and expressed more collagen III and some other proteins seen in the tendinopathic tendons.

Augusta D. Mazzocca et al (16) evaluated changes of the biceps tendon in three groups; biceps tendon instability-patients, degenerative joint disease and tendinosis. They compared the proximal (intraarticular) and distal (extra-articular) regions of the tendons using several histomorphologic and molecular approaches. Immunohistochemistry, proteoglycan analysis, polarized light analysis, and protein molecular analysis were combined for this purpose. The proximal (intra-articular) segment of the LHB showed more signs of degenerative changes than the distal (extra-articular) portion throughout all diagnostic groups. The tendon instability group showed the greatest degree of degenerative changes in the proximal LHB, most likely because more strain on the tendon from the support slings and other surrounding tissue. A fourth control group, fresh frozen healthy tendons, had significantly lesser changes in the LHB-tendon.

In our material, we also have found that all seven tendons have histological changes in the intraarticular part of the LHB-tendon (fig 1). The same is also evident in the 7-Tesla MRI. The macroscopic changes that Pascal Boileau describes (14), are by our experimental study shown microscopically. The more the fatty infiltration, degeneration and structural changes, the greater is the difference between the diameter 
of the intraarticular and sulcus part of the LHB-tendon. If it is possible to measure this diameters of the LHB-tendon on the routine preoperative MRI-scanning, this measurements can be used as a guideline for planning the procedure; the greater the difference, the more likely is the biceps involved in the pathology giving rise to the anterior shoulder pain, and a tenodesis or tenotomy must be planned. In Norway, the MRI-scanners in use are 1.5 Tesla-scanners, and it is not possible to do these measurements on these machines.

The finding of increased fatty infiltration and degeneration with increasing difference of the intraarticular and sulcus diameter also may be an indication that the autotenodesis after a tenotomy may give postoperative pain due to a possible inflammation around the necrotic and degenerative LHB-tenotomyarea. According to these findings, the subpectoral tenodesis with removal of the intraarticular portion of the LHB-tendon is a theoretical better procedure than the simple tenotomy also in the middle-aged patients. In our department, we also observe that most of the revisions after LHB-procedures are after LHB-tenotomies.

Conclusion: In all our patients, the intraarticular part of the LHB-tendon showed degeneration and increased diameter, both in peroperative findings, 7 Tesla MRI and histology. This give entrapment of the tendon in the early proximal part of the sulcus and because of this, anterior shoulder pain. We have with our experimental study, shown a pathological-anatomical correlate to the anterior shoulder pain. It is important to remove the intraarticular part of the tendon, and a subpectoral biceps tenodesis with removal of the proximal part of the tendon is thereby the treatment of choice in these patients. These changes could not be visualizes on the routine 1.5 Tesla MRI scanning and the 1.5 Tesla MRI is not a good diagnostic tool for the patients with anterior shoulder pain. We have also constructed a grading system of LHB-tendon degeneration that will be useful in the clinical decisionmaking

\section{Results}

Our experimental study showed that all the degenerative changes in the LHB-tendon were in the intraarticular part of the tendon (I), not in the intertubercular sulcus (S). We also found in both the histological examination and the 7-Tesla MRI that the diameter of the tendon is greater in the intraarticular part than in the intertubercular sulcus part. We may thus state that the more degeneration, the greater the difference, and the greater the ratio diameter $\mathrm{I} /$ diameter $\mathrm{S}$ (Table 2). Because of this, the more degeneration of the tendon, the more wedge-shaped is the transitional zone between the intraarticular and sulcus part of the tendon. 
Figure 1 illustrates changes on the histology and seven Tesla MRI-scanning in minimal, moderate and severe degeneration of the biceps tendon. The intratendinous structure was preserved in the intertubercular sulcus part of the tendon, but fibrosis ant fatty infiltration can also be seen in the grade 3 severe degenerated LHB-tendon in the intertubercular sulcus part of the tendon.

Figure 2 illustrates the changes in preoperative diagnostic 1.5 Tesla MRI, peroperative finding, histology and 7Tesla postoperative MRI scanning of one patient with grade 2 moderate degenerative changes in the LHB-tendon.

None of the peropertive 1.5 Tesla MRI-scannings gave us information about the LHB-tendon that we could use in the clinical decision-making.

\section{Discussion}

In our opinion, our results should alter the surgical practice in the surgical treatment of degenerative changes and pain in the LHB-tendon. Today we have two different procedures; biceps tenodesis and biceps tenotomy. Our results suggest that the subpectoral biceps tenodesis with removal of the intraarticular part of the tendon is the surgical treatment of choice when there is peroperative findings that suggest degeneration of the LHB-tendon.

We have found by our combination of MRI - and histological studies that close to all pathology in the LHB-tendon are located in the intraarticular part of the tendon, with degeneration, fibrosis and fatty infiltration. This implies that it is the intraarticular part of the tendon, and not the part situated in the sulcus that gives the patient the LHBtendon mediated shoulder pain. By doing a simple tenotomy the wedge, shaped LHB-tendon will stay in the intertubercular sulcus and cause inflammation and necrosis of the degenerated LHB-tendon, with further anterior pain in the shoulder after the surgery. Further, on we have shown that MRI may demonstrate LHB-tendon pathology preoperatively better than it does today, and that none of the preoperative MRIs in our experimental study gave us important information of the tendon.

One of the weaknesses of our study is that we have a small material, but the strength is that all the tendons show that it is the intraarticular part of the tendon that is degenerated.

Gill et al reported pain relief in $96,7 \%$, return to sport in $90 \%$ and return to work in $96,7 \%$ after an isolated arthroscopic bicepstenotomy in a group of 30 patients, but also a complication rate of $13.3 \%$ (pain with activity, cosmetic deformity) in 4 of the 30 patients (5). Walch et al reported a $87 \%$ success rate after arthroscopic bicepstenotomy in patients with irreparable rotator cuff tears, emphasizing the role of the biceps as a pain generator in the shoulder and the ability to alleviate pain by its removal from the glenohumeral joint (8).

Heckman et al (13) review in their article the treatment of LHB-tendon pathology and indications for revision of tenodesis and tenotomies. It is common with pain, muscle cramps and cosmetic problems 
after biceps tenotomies. Subpectoral bicepstenodesis and completely removing the tendon from the sulcus, as a method of revision of failed LHB-tenotomies, is the treatment of choice.

Pascal Boileau et al (14) described in 2004 a macroscopic hypertrophy of the LHB-tendon as a mechanical condition that entrap the tendon within the joint and locking the arm on elevation. The patients presented with anterior pain and loss of active and passive elevation averaging 10-20 degrees (14). Because of this hypertrophy, the tendon is unable to slide into the bicipital groove during elevation. The complete and symmetrical elevation was restored after resection of the intraarticular portion of the LHB-tendon.

Michael Joseph et al (15) did a histological and molecular characterization of the intraarticular and extraarticular portion of the post tenotomi LHB-tendon in patients with LHB-tendinopathy. The intraarticular tendon exhibited histological characteristics of tendinopathy, compared with the extra-articular portion, had structural disorganization and expressed more collagen III and some other proteins seen in the tendinopathic tendons.

Augusta D. Mazzocca et al (16) evaluated changes of the biceps tendon in three groups; biceps tendon instability-patients, degenerative joint disease and tendinosis. They compared the proximal (intraarticular) and distal (extra-articular) regions of the tendons using several histomorphologic and molecular approaches. Immunohistochemistry, proteoglycan analysis, polarized light analysis, and protein molecular analysis were combined for this purpose. The proximal (intra-articular) segment of the LHB showed more signs of degenerative changes than the distal (extra-articular) portion throughout all diagnostic groups. The tendon instability group showed the greatest degree of degenerative changes in the proximal LHB, most likely because more strain on the tendon from the support slings and other surrounding tissue. A fourth control group, fresh frozen healthy tendons, had significantly lesser changes in the LHB-tendon.

In our material, we also have found that all seven tendons have histological changes in the intraarticular part of the LHB-tendon (fig 1). The same is also evident in the 7-Tesla MRI. The macroscopic changes that Pascal Boileau describes (14), are by our experimental study shown microscopically. The more the fatty infiltration, degeneration and structural changes, the greater is the difference between the diameter of the intraarticular and sulcus part of the LHB-tendon. If it is possible to measure this diameters of the LHB-tendon on the routine preoperative MRI-scanning, this measurements can be used as a guideline for planning the procedure; the greater the difference, the more likely is the biceps involved in the pathology giving rise to the anterior shoulder pain, and a tenodesis or tenotomy must be planned. In Norway, the MRI-scanners in use are 1.5 Tesla-scanners, and it is not possible to do these measurements on these machines.

The finding of increased fatty infiltration and degeneration with increasing difference of the intraarticular and sulcus diameter also may be an indication that the autotenodesis after a tenotomy may give postoperative pain due to a possible inflammation around the necrotic and degenerative LHB-tenotomyarea. According to these findings, the subpectoral tenodesis with removal of the intraarticular portion of the LHB-tendon is a theoretical better procedure than the simple tenotomy also in the middle-aged patients. In our department, we also observe that most of the revisions after LHB-procedures are after LHB-tenotomies. 


\section{Conclusions}

In all our patients, the intraarticular part of the LHB-tendon showed degeneration and increased diameter, both in peroperative findings, 7 Tesla MRI and histology. This give entrapment of the tendon in the early proximal part of the sulcus and because of this, anterior shoulder pain. We have with our experimental study, shown a pathological-anatomical correlate to the anterior shoulder pain. It is important to remove the intraarticular part of the tendon, and a subpectoral biceps tenodesis with removal of the proximal part of the tendon is thereby the treatment of choice in these patients. These changes could not be visualizes on the routine 1.5 Tesla MRI scanning and the 1.5 Tesla MRI is not a good diagnostic tool for the patients with anterior shoulder pain. We have also constructed a grading system of LHB-tendon degeneration that will be useful in the clinical decisionmaking

\section{Declarations}

Ethics approval and consent to participate Consent for publication Availability of data and material Competing interests

- The study was approved by the Regional Ethics Committee of Western Norway.

- All patients gave a written consent of participation in the study and the use of data for publication; after been given a written description of the study, and an oral information about both the study and that it was intended for publication.

- No author have competing interests. None of the material are in use for other purposes.

- The data and materials may be obtained from the authors (except direct links to patient journals). We have consent from the patients for using and storing data.

Funding

- The experimental part of the study was partly funded by West Norwegian Health authorities

Authors' contributions

- Both authors were very involved in all parts of the study.

- KH did the clinical examinations and the operations. JTG did the MRI analyses and the design of the study. Both authors did all analyses and writing of the article together.

Acknowledgements

- Acknowledgements: Tina Pavlin PhD for help with the 7-Tesla MRI-performance. The 7-Tesla MRI and the histological slices were done at the Institute of Basic Medical Science, University of Bergen. The clinical part of the study was performed at Haraldsplass Deaconess Hospital, Bergen.

Author information 
- $\mathrm{KH}$ has a long career in orthopaedics and is now a senior consultant of orthopaedic surgery. He has taken a special interest in treatment of the shoulder. JTG is a professor of radiology and has a broad background in all areas of radiology. He has earlier performed both clinical and experimental studies on MRI.

- Abbreviations

- LHB: Long Head Biceps muscle (the biceps muscle's long head)

- MRI: Magnetic Resonance Imaging

- T: Tesla; SI unit for a derived unit of the magnetic induction

- H+E staining: Hematoxylin and eosin stain

- TIVA: Patient in total anaesthesia

- RF: Radiofrequency pulse in the MRI

- TR: Time to repetition (MRI)

- TE: Time to echo (MRI)

\section{References}

1. Nicholas R. Slenker, Kevin Lawson, Paul H. Marks et al, Biceps TEnotomy Versus Tenodesis:Clinica outcomes. Arthroscopy; The Journal of Arthroscopic and Related Surgery. Vol 28, No 4(April), 2012: pp 576-582

2. Heng `an Ge, Quiang Zhang, Yeqing Sun $Y$ et al, Tenotomy or Tenodesis for the Long Head of Biceps Lesions in Shoulders: A Systematic Review and Meta-Analysis. PLOS ONE 2015;10(3):1-11

3. Levy DM, Meyer ZI, Campbell KM et al, Subpectoral Biceps Tenodesis. Am J Orthop. 2016 February; 45(2):68-74

4. Heckman DS, Creighton RA, Romeo AA. Management of failed biceps tenodesis or tenotomy:Causes and treatment. Sports Med Arthrosc Rev. 2010 Sep;18:173-180

5. Augustus D Mazzocca, Anthony A. Romeo. Operative techinques in Sports Medicine, vol 11, Issue 1, January 2003, Pages 6-14

6. William F. Scully, David J. Wilson, Jason A. Grassbaugh. A Simple Surgical technique for Subpectoral Biceps Tenodesis Using a Doulble_Loaded Suture Anchor. Arthroscopic Tecniques Vol 2, No 2(May), 2013;pp e191-e196

7. Jonathan F. Dickens, Kilcoyne KG, Trintle SM et al. Subpectoral Biceps tenodesis,. An anatomic Study an Evaluation of At-Risk Structures. The American Journal of Sports medicine 2012 okt;40(10:233741.

8. Gilles Walch, T. Bradley Edwards Aziz Boulahia et al. Arthroscopic tenotomy of the long head of the biceps in the treatment of rotator cuff tears; clinical and radiological results in 307 cases. J Shoulder Elbow surg. May/June 2005; Volume 14, number 3:238-246. 
9. Thomas J Gill, Elisabeth Mclrvin, Scott D. Mair et al, Results of biceps tenotomy for treatment of pathology of the long head of the biceps brachii. J Shoulder Elbow surg 2001;Vol 10 number 3:247249.

10. Andrew R. Hsu, Neil S Ghodadra, Mathew T. Provencher et al. Biceps tenotomy versus tenodesis: a rewiev of clinical outcomes and biomechanical results. Journal of Shoulder and Elbow Surgery 2011; 20:326-332

11. Fuchs $B$, Weishaupt $D$, Zanetti $M$ et al. Fatty degeneration of the muscles of the rotator cuff: assessment bycomputed tomography versus magnetic resonance imaging. J Shoulder Elbow Surg. 1999;8:599-605.

12. Jeremy S Somerson, Jason E. Hsu, Jacob D. Gorbathy et al, Classification in brief; Gautallier Classification of Fatty Infiltration of the Rotator Cuff Muscle. Clin Orthop. Relat Research 2016; 474:1328-1332

13. Daniel S. Heckman, R. Alexander Creighton, Anton A. Romero. Management of Failed Bicepstenodesis or Tenotomy; Causation and Treatment. Sports Med Arthrosc Rev.;Volume 18, Number 3, September 2010:173-180.

14. Pascal Boileau, Philip M. Arens, Armodios M. Hatzidakis. Entrapment of the long head of the biceps tendon: The hourglass biceps-A cause of pain and locking of the shoulder. J Shoulder Elbow Surg 2004; Volume 13, Number 3:249-257.

15. Michael Joseph, Carl M. Maresh, Mary Beth McCarthy et al. Histological and Molecular Analysis of the Biceps Tendon Long Head Post Tenotomy. Journal of Orthopaedic Research 2009;27:1379-1385

16. Augusta D Macocca, Mary Beth McCarthy, Felicia A Ledgard et al. Histomorphologic Changes of the Long Head of the Biceps Tendon in Common Choulder Pathologies. Arthroscopy; The Journal of Arthroscopic and Related Surgery; Vol 29, No 6 2013:972-981

17. Bennett WF. Specificity of the Speed's test: arthroscopic techniquefor evaluating the biceps tendon at the level of the bicipital groove. Arthroscopy. 1998;14:789-796.

18. O'Brien SJ, Pagnani MJ, Fealy S. et al. The active compression test: a new and effective test for diagnosing labral tears and acromioclavicular joint abnormality. Am J Sports Med. 1998;26:610-613.

\section{Tables}

Table 1 Staging of the biceps tendon regarding peroperative visual findings, histology of the tendons and 7 Tesla MRI scanning of the tendons. ASS (anterior support sling). PSS (posterior support sling). 


\begin{tabular}{|c|c|c|c|}
\hline Tendon & Peroperative findings & Histology & 7 Tesla MRI \\
\hline Normal & $\begin{array}{l}\text { Normal tendon, no teno- } \\
\text { synovitis, no stiffness, intact } \\
\text { anterior and superior } \\
\text { support-slings }\end{array}$ & $\begin{array}{l}\text { No changes in structure, } \\
\text { intact fibers, no fatty } \\
\text { infiltration between fibers. No } \\
\text { caliber differense intra/sulcus }\end{array}$ & $\begin{array}{l}\text { No changes in structure, intact } \\
\text { fibers, no fat signal between } \\
\text { fibers, no caliber difference } \\
\text { intraart./sulcus }\end{array}$ \\
\hline $\begin{array}{l}\text { Minimal } \\
\text { degeneration } \\
\text { (Grade 1) }\end{array}$ & $\begin{array}{l}\text { Slight stiffness and } \\
\text { tenosynovitis, slight de- } \\
\text { generation with fib-rillation } \\
\text { at the tendon origin/labrum. } \\
\text { ASS/PSS intact }\end{array}$ & $\begin{array}{l}\text { Intraarticular changes in } \\
\text { structure, none or slightly } \\
\text { visible fatty infiltration } \\
\text { between fibers. Normal } \\
\text { structure sulcus part. } \\
\text { Different diameters intra and } \\
\text { sulcus. }\end{array}$ & $\begin{array}{l}\text { Intraarticular changes in } \\
\text { structure, slight fatty signal } \\
\text { between fibers Normal } \\
\text { structure sulcus part. Different } \\
\text { diameter intra/sulcus }\end{array}$ \\
\hline $\begin{array}{l}\text { Moderate } \\
\text { degeneration } \\
\text { (Grade 2) }\end{array}$ & $\begin{array}{l}\text { Intraart. stiffness and } \\
\text { tenosynovitis. Yellowish } \\
\text { colour as a sign of intraart. } \\
\text { degeneration. Increasing } \\
\text { degeneration of origin. } \\
\text { Distended ASS and PSS } \\
\text { with synovitis and } \\
\text { fibrillation. No subluxation }\end{array}$ & $\begin{array}{l}\text { Intraart changes with up to } \\
50 \% \text { fibers } / 50 \% \text { fatty } \\
\text { infiltration. Intact fiber } \\
\text { structure.Intact } \quad \text { sulcus } \\
\text { tendon. Increasing difference } \\
\text { in diameter between intraart.- } \\
\text { sulcus part. }\end{array}$ & $\begin{array}{l}\text { Intraart changes with up to } \\
50 \% \text { fibers/50 \% fatty } \\
\text { infiltration. Intact fiber } \\
\text { structure.Intact sulcus tendon. } \\
\text { Increasing difference in } \\
\text { diameter between intraart.- } \\
\text { sulcus part }\end{array}$ \\
\hline $\begin{array}{l}\text { Severe } \\
\text { degeneration } \\
\text { (Grade 3) }\end{array}$ & $\begin{array}{l}\text { Stiffness, tenosynovitis, } \\
\text { yellowich colour, visible } \\
\text { structural damage of } \\
\text { tendon, ASS/PSS not intact, } \\
\text { and tendon can be luxated }\end{array}$ & $\begin{array}{l}\text { Intraart. changes with more } \\
\text { than } 50 \% \text { fatty infiltration, } \\
\text { altered } \\
\text { structure,vacuolisation } \\
\text { increasing changes in sulcus } \\
\text { part with fatty infiltration. } \\
\text { Increasing difference in } \\
\text { diameter between intraart- } \\
\text { sulcus part }\end{array}$ & $\begin{array}{l}\text { Intraart. changes with more } \\
\text { than } 50 \% \text { fatty infiltration, } \\
\text { altered fiber structure, } \\
\text { vacuolisation } \\
\text { changes in sulcus part with } \\
\text { fatty infiltration. Increasing } \\
\text { difference in diameter between } \\
\text { intraart-sulcus part }\end{array}$ \\
\hline
\end{tabular}

Table 2: Gender, age, peropertive, MRI and histological classifications (minimal, moderate and severe degeneration) and ratio between the intraarticular and sulcus diameter in the MRI-scanning and histology in the 7 tendons in this experimental study. The patients were from 31-47 years of age, 4 females and 3 males.

\begin{tabular}{|l|l|l|l|l|l|}
\hline Tendon number & Perop. classification & 7-Tesla & 7-tesla I/S ratio & Histologi & Histologi I/S ratio \\
\hline 1 & Minimal & Moderate & 1,3 & Moderate & 1,2 \\
\hline 2 & Severe & Severe & 2,3 & Severe & 2,2 \\
\hline 3 & Moderate & Severe & 1,3 & Moderate & 1,4 \\
\hline 4 & Moderate & Mild & 1,3 & Moderate & 1,5 \\
\hline 5 & Moderate & Severe & 1,6 & Severe & 1,7 \\
\hline 6 & Moderate & Moderate & 1,4 & Moderate & 1,4 \\
\hline 7 & Minimal & Mild & 1,1 & Moderate & 1,2 \\
\hline
\end{tabular}

\section{Abbreviations}

LHB: Long Head Biceps muscle (the biceps muscle's long head) 
MRI: Magnetic Resonance Imaging

T: Tesla; SI unit for a derived unit of the magnetic induction

$\mathrm{H}+\mathrm{E}$ staining: Hematoxylin and eosin stain

TIVA: Patient in total anaesthesia

RF: Radiofrequency pulse in the MRI

TR: Time to repetition (MRI)

TE: Time to echo (MRI)

\section{Figures}
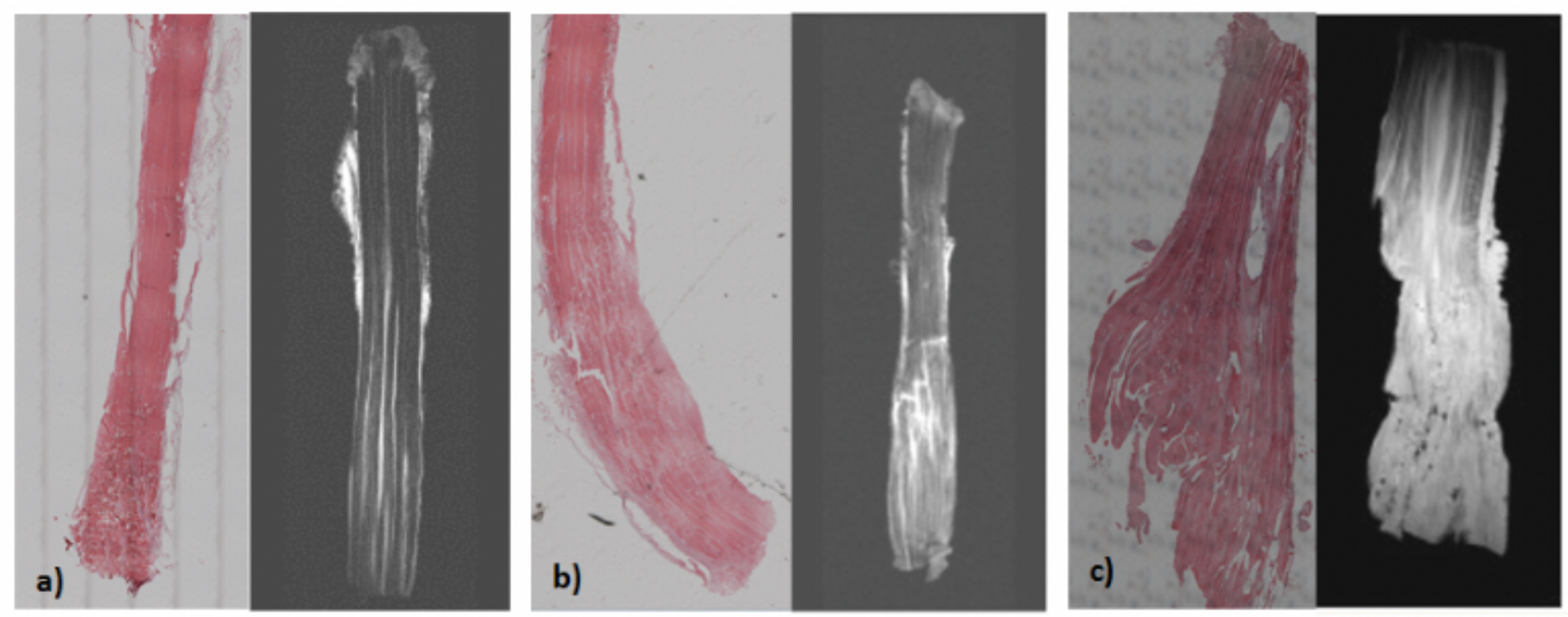

Figure 1: Histology and 7 Tesla MRI midsubstance longitudinal sections of a) grade 1 altered, b) grade 2 altered and c) grade 3 altered biceps caput longum tendons. Articular part down in the figure.

\section{Figure 1}




\section{Figure 2}
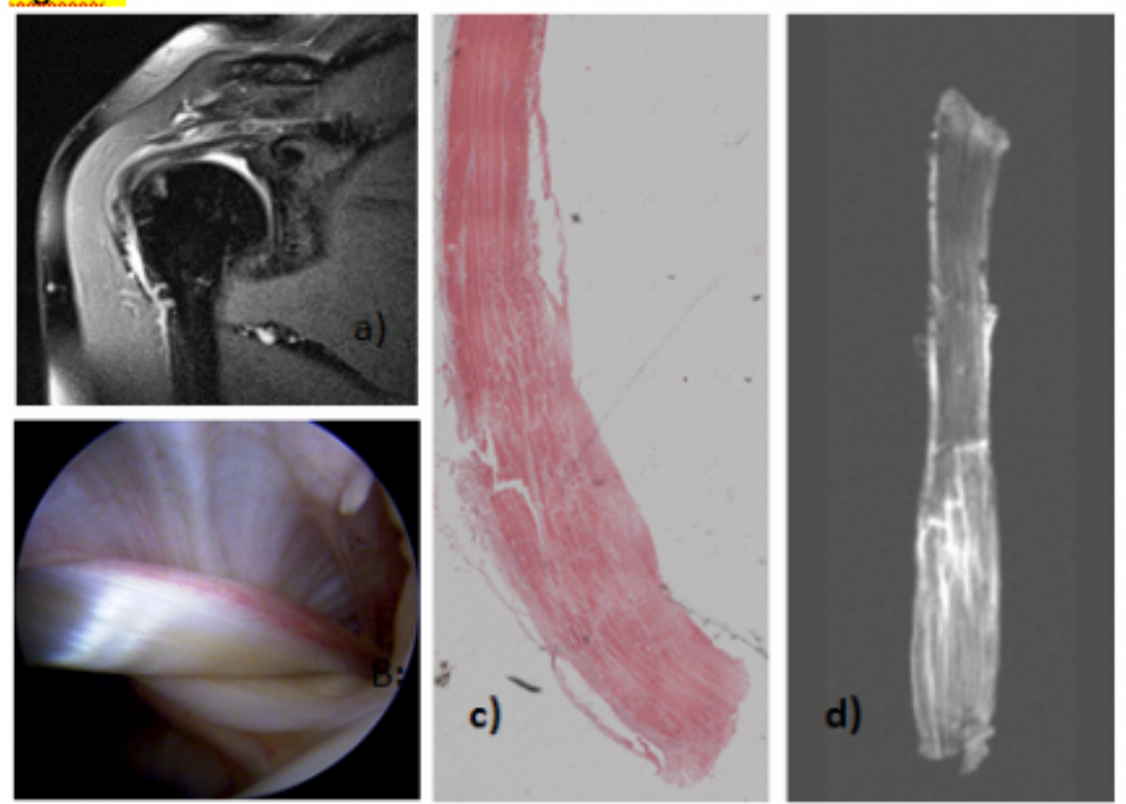

Preoperative 1.5 T MRI T2 coronal section (a), peroperative arthroscopic view of the long head of the biceps tendon in the joint (b), histology c), and experimental 7T MRI of the tendon (d). Articular part down in (c) and (d).

\section{Figure 2}

